\title{
Asesoramiento en la Evaluación del Proceso de Aprendizaje al alumnado de Secundaria
}

\section{Counseling the Evaluation of the Learning Process in Secondary Education Students}

\author{
Eufrasio PÉREZ NAVÍO \\ Universidad de Jaén
}

Recibido: Septiembre 2013

Aceptado: Noviembre 2013

\section{Resumen}

En el presente artículo se pone de relieve las inquietudes que, el alumnado del Instituto de Educación Secundaria "María Bellido" de Bailén y del Instituto de Educación Secundaria "Jabalcuz" de Jaén (España), tienen de la organización y planificación de la evaluación que realiza el profesorado que imparte docencia a todos los cursos de primero de Bachillerato.

Para ello, se ha desarrollado un cuestionario ad hoc que nos ha permitido conocer la valoración que realizan de la evaluación que llevan a cabo el profesorado. El instrumento consta de treinta y tres preguntas, nueve abiertas y el resto cerradas, divididas en siete dimensiones: 1 . Concepto de evaluación/Concepto de evaluación del docente, 2. Instrumentos de evaluación, 3. Sistemas metodológicos, 4. Relación con el aula, 5. Momento de evaluación, 6. Asesoramiento del docente y 7. Habilidades del docente.

En general, la investigación realizada nos arroja que el alumnado sigue teniendo desasosiego a la evaluación. Es percibida, en la mayoría de los casos, como influencia negativa en su vida académica.

Palabras clave: secundaria, evaluación, aprendizaje, docente, estudiante, asesoramiento.

\begin{abstract}
This article highlights the concerns that students of Bailen Secondary School "Maria Bellido" and Jaen Secondary School "Jabalcuz" (Spain), have about the organization and planning of the evaluation process done by teachers who are teaching all first Baccalaureate courses.

To this end, it has been developed an ad hoc questionnaire that has allowed us to get to know the assessment done about the evaluation being conducted by teachers. The instrument consists of thirty-three questions, nine of which are open, being the rest closed, and have been divided into seven dimensions: 1. Evaluation concept/Teacher evaluation concept, 2. Assessment instruments, 3. Methodological Systems, 4. Relationship to the classroom, 5. Assessment time, 6. Teacher counseling and 7. Teaching skills. In general, the investigation shows that students' still have a sense of uneasiness towards the evaluation process. It is sensed, in most of the cases, as a negative influence in their academic life.
\end{abstract}

Keywords: secondary, assessment, learning, teacher, student, counseling, evaluation. 
La evaluación educativa considera a las instituciones educativas como una unidad básica y fundamental de cambio e innovación en educación; este cambio implica la asunción por parte de toda la comunidad educativa, pero no debemos caer en el error de considerar a todas las instituciones educativas iguales y, sobre todo, que tengan los mismos problemas de evaluación, susceptibles además de soluciones rápidas y uniformes.

La evaluación está íntimamente ligada con la toma de decisiones, porque al fin y al cabo evaluar supone decidir. Actualmente no existe un modelo claro que asesore al profesorado en tareas de evaluación de los discentes, quizás debido al gran tabú o respeto que siempre se ha tenido al evaluar a los profesionales de la educación. No obstante, Medina (2013) o Villar y Alegre (2012) han señalado aspectos importantes referidos a este asunto que a lo largo del artículo iremos descubriendo.

El objetivo fundamental de la evaluación es la mejora de lo que evaluamos, lo que implica dar mayor servicio o calidad a ese aspecto. Pero una dimensión de la calidad es la eficacia con que se haga esa evaluación para que sea como la pescadilla que se muerde la cola y funcione como un feedback del proceso.

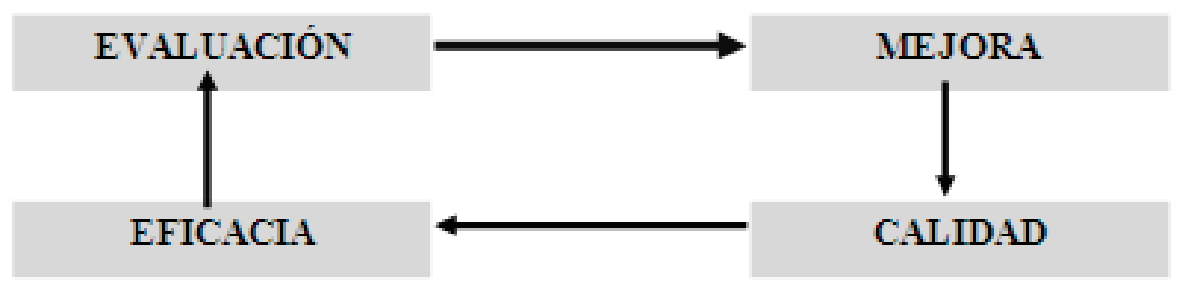

Tabla 1. Proceso de Evaluación (Fuente: Elaboración propia)

Según esta Tabla 1, calidad, eficacia, conocimiento profundo, incidencias en la mejora y evaluación deben de estar ligadas para que exista una buena conexión de interrelación y se mejore, en este caso de estudio, la evaluación de los docentes hacia sus discentes.

El apoyo o asesoramiento al profesorado en la tarea evaluadora es un fenómeno relativamente novedoso en el ámbito educativo. A la ausencia de una tradición investigadora hay que añadir la desestructuración e indefinición que presenta. Por ello, el presente artículo muestra las opiniones de 128 estudiantes de dos importantes institutos de educación secundaria de dos localidades distintas de la provincia de Jaén, sobre cómo perciben la evaluación que su profesorado realiza con ellos, qué mejorar y cómo asesorar al profesorado para que mejore la evaluación.

Entendemos el asesoramiento como el proceso de ayuda, tanto interno como externo, que se facilita al profesorado, para que a través de la reflexión personal se reconozcan las posibilidades de cambio en centros, se diseñen y se lleven a la práctica en el aula. Villar (1992:6) en el prólogo a la obra "Desarrollo profesional centrado en la escuela", corrobora esta idea, cuando afirma: "La idea básica sobre el perfil del profesor se refiere a la reflexivilidad del docente (...) El desarrollo profesional 
previsto para los profesores es concomitante con una mejora de la institución escolar". Pero no se entiende el asesoramiento si el docente no adopta el compromiso de valorar el centro como estructura organizativa de colaboración, cuyo conocimiento y autorrevisión constituyen el núcleo del cambio como mejora. Es por ello que se considera el desarrollo basado en la escuela como espacio para el asesoramiento, que facilita el cambio e innovación educativa en y desde los centros escolares.

Se plantea como objetivo: conocer cuál es la manera o forma de evaluar que tiene el profesorado de secundaria, así como la interpretación que dan los discentes de la forma de evaluación que les hacen sus docentes.

Desde este trabajo intentamos ofrecer al alumnado y profesorado un escenario que le ayude a reflexionar, conocer, analizar, aplicar y desarrollar de forma responsable y autónoma la evaluación como estimulo de calidad realizado a través del asesoramiento.

La evaluación del discente en los procesos de aprendizaje y del rendimiento requiere de un meticuloso conocimiento de las actuaciones y de los datos más significativos como contenidos personales que cada estudiante aprende desde su potencialidad intelectual, socio-emocional y física, enriquecida o inhibida por los múltiples contextos socio-culturales y las interacciones con el medio escolar.

La valoración de los complejos procesos educativos y del rendimiento académico exige del profesorado una capacidad y preparación en consonancia con tal complejidad.

Esta complejidad no es ajena a la auto y coevaluación del profesorado, planteándose a cada docente la comprensión, análisis y toma de decisiones para valorar la coherencia entre lo previsto $\mathrm{y}$ realmente realizado en los procesos de enseñanza. La autoevaluación requiere de un saber globalizado y de una indagación creadora para valorar en todas sus dimensiones la tarea formativa de enseñar, en consonancia con esta acción personal y colaborativa, se logrará que cada profesional sitúe en toda su complejidad los procesos de enseñanza - aprendizaje de los estudiantes.

La evaluación en sus diversas modalidades: diagnóstico - estimativa, formativo procesual y contrastadora - final nos ofrece sus etapas que junto a la variedad y diferenciación de cada estudiante, contexto y elementos evaluados ponen de manifiesto que es una de las tareas más implicadoras del profesorado.

La concepción y el sentido de la formación y mejora que acompaña a la autoevaluación servirá a los docentes para crear situaciones y criterios claros que posibiliten a los estudiantes avanzar en la autoevaluación de las tareas y procesos, a la vez que el estilo de coevaluación entre colegas apoyará esta práctica evaluadora entre los estudiantes, aplicándose con modelos complementarios y formativos de evaluación externa.

¿Cómo plantear esta transferencia?, ¿Cómo elaborar las técnicas y métodos más pertinentes?, ¿Cómo crear esta cultura de la reciprocidad y el análisis coevaluador?.

La transferencia se basa en el conocimiento que adquiere el profesorado del saber y práctica evaluadora. El desarrollo de actitudes favorables a la autoevaluación lleva a 
cada docente a proyectar este modelo en la mejora de los procesos de aprendizaje y en la participación responsable de los estudiantes en esta modalidad evaluativa.

Hemos de integrar nuestra experiencia biográfica y en el estilo evaluador del docente las posibilidades y limitaciones de esta concepción para que se alcance esta opción evaluadora.

Un buen docente ha de ser un esmerado evaluador de su alumnado sin limitarse exclusivamente al tradicional examen o control realizado sobre el estudiante, sino que debe evaluarse con la mayor objetividad posible y con la mayor amplitud de aspectos que integran el proceso de enseñanza - aprendizaje y el contexto educativo.

Tradicionalmente las tareas de evaluación se han centrado en torno a una finalidad prioritaria: valorar los resultados del aprendizaje del alumnado con vistas a poner una calificación. Sin embargo, la práctica evaluadora debe responder a diversas finalidades: ayudar a los estudiantes a avanzar en su proceso de aprendizaje; comprobar y hacer balance de los conocimientos adquiridos; informar a familias y alumnos/as de los resultados alcanzados al final de un período determinado, etc.

No hay que olvidar que los procesos de enseñanza y de aprendizaje son interdependientes, y que por tanto, son igualmente objeto de evaluación, este artículo se centra en el proceso de aprendizaje.

En el análisis sincrónico de la Reforma Educativa en la Educación Secundaria Obligatoria (Rodríguez Diéguez y otros, 1997) se aplica un conjunto de encuestas. Una de las cuestiones presentadas con relación a la evaluación es la siguiente:

Indique la importancia que concede a cada uno de los siguientes procedimientos e instrumentos de evaluación para su área y en Educación Secundaria:

- Observación de los trabajos que el alumno realiza en clase.

- Observación de los cuadernos.

- Análisis de los trabajos realizados en casa.

- Exámenes orales.

- Exámenes escritos consistentes en varias preguntas cortas.

- Examen escrito sobre un tema.

- Prueba objetiva.

- Examen con material.

- Escalas de observación de los aprendizajes individuales para cada uno de los objetivos pormenorizados.

- Observación de las intervenciones orales de los alumnos en clase y con su grupo.

- Autoevaluación del alumnado.

- Preguntas en clase.

Destaca la utilización de técnicas informales de evaluación, de utilización más fácil y cómoda, y que además se encuentran en una relación más directa con la evaluación predominantemente positiva, "por imperativo legal". Las cinco técnicas ordenadas de más a menos importancia que le concede el profesorado son las siguientes: 
- Trabajo en clase.

- Cuadernos.

- Observación.

- Preguntas.

- Exámenes con preguntas cortas.

Al abordar el trascendental papel que le corresponde al docente en el desarrollo de la evaluación, se corre el peligro de caer en tópicos o en insinuaciones acusatorias, queriendo poner de manifiesto "lo malos" que son los docentes, por lo mal que desempeñan la función evaluadora.

La función del evaluador no es una nueva tarea que la L.O.G.S.E., la L.O.C.E. o la L.O.E. adjudica al docente. Dicha función era ya asumida y ejercida con anterioridad. Pero no cabe duda que las leyes educativas han servido para reforzar con una nueva dimensión la tarea de evaluar. El carácter formativo de la evaluación, que determina todo el proceso de aprendizaje, es probablemente el primer y principal rasgo que define esta nueva realidad.

Es relativamente fácil poner de relieve el comportamiento docente en relación con la práctica de la evaluación ahora, conforme a los nuevos planteamientos, cuando se vive inmerso en una cultura, de muchos años, donde lo determinante era el examen y las calificaciones o notas, sin que entre tanto haya mediado una formación específica del profesorado. Sin embargo, corresponde al profesorado ser los impulsores del cambio hacia una nueva cultura evaluadora, donde la persona del discente es más importante que la calificación de su rendimiento.

La evaluación es siempre la misma y es el profesorado el que solicita unas finalidades y unas funciones determinadas, según el momento, y demás circunstancias que rodean a una intervención didáctica. El profesor o la profesora es la mejor persona situada para generar el cambio de cultura evaluadora, en el día a día del aula.

El profesorado se encuentra con que la Administración Educativa y la sociedad le piden numerosas tareas, más y mejores maneras en su desempeño docente, que le sumergen en la inseguridad, y que, finalmente, se decanta en ansiedad o impotencia.

El papel del docente en el aula es también otro factor de inquietud. Su papel de sujeto agente (el profesor enseña), se ha adjudicado al estudiante (el discente aprende). $\mathrm{Y}$ para definir su tarea vuelven a aparecer otros nombres: el docente es asesor, motivador, animador, programador, evaluador, orientador, entrenador, conductor, facilitador, amigo líder, entrenador de personas, etc., en definitiva, se puede considerar a un docente como el coach de un estudiante (Pérez, 2013).

Pero además, si la educación tiene como finalidad el desarrollo de la persona humana y su integración en la sociedad, es evidente que, ante un reto como éste, hay que tomar precauciones. La práctica de la evaluación es, de hecho, la concreción de estas precauciones. 
Es preciso que, durante el proceso de aprendizaje, las personas implicadas puedan apreciar la eficacia de su trabajo para poder hacer las rectificaciones oportunas en el caso de que se presenten desviaciones significativas respecto a las finalidades deseables. El docente, por su preparación, por su implicación y por la posibilidad que tiene de actuar en el proceso, es la persona idónea para ejercer el papel preponderante de evaluador. Programar, entrenar y evaluar son tres funciones básicas que perfila el papel del profesor.

En la educación secundaria la evaluación tiene una intencionalidad formativa y se puede añadir una intención orientadora hacia la vocación y la futura profesión.

La nueva cultura evaluadora debe expresar con hechos significativos que evaluar no es someter al alumnado cada cierto tiempo a un examen para adjudicarles una nota que les informe del nivel alcanzado, sino una actitud de observación, de recogida de datos y de análisis que permita al docente, y a las demás personas implicadas en el proceso, percibir, lo antes posible, las capacidades de los estudiantes y las disfunciones de la acción educativa. Es de gran utilidad tener un planteamiento sinóptico de la evaluación como estrategia de representación gráfica que pone de manifiesto aspectos estrechamente relacionados entre sí en los procesos de la enseñanza-aprendizaje y de la evaluación.

\begin{tabular}{|c|c|c|c|}
\hline & \multicolumn{2}{|c|}{ ENSEÑANZA } & APRENDIZAJE \\
\hline \multirow{4}{*}{$\begin{array}{l}\text { - Comunicación. } \\
\text { - Metodología. } \\
\text { - Innovación. } \\
\text { - Facilitación. }\end{array}$} & \multicolumn{2}{|l|}{ EVALUACION } & \multirow{3}{*}{$\begin{array}{l}\text { Interés/motivación. } \\
\text { Trabajo personal. } \\
\text { Aprovechamiento } \\
\text { en clase. } \\
\text { Rendimiento } \\
\text { académico. }\end{array}$} \\
\hline & $\begin{array}{l}\text { Actitud personal. } \\
\text { Diseño del currículum del } \\
\text { aula. } \\
\text { Explicación/comunicación. } \\
\text { Estrategias de Enseñanza. } \\
\text { Proceso de enseñanza. } \\
\text { Resultados de la enseñanza. }\end{array}$ & $\begin{array}{l}\text { Disposición personal. } \\
\text { Programación } \\
\text { individual/colectiva. } \\
\text { Comprensión/ritmo de } \\
\text { aprendizaje. } \\
\text { Procedimientos de } \\
\text { aprendizaje. } \\
\text { Proceso de aprendizaje. } \\
\text { Logros de aprendizaje. }\end{array}$ & \\
\hline & \multicolumn{2}{|c|}{ AUTOEVALUACIÓN } & \\
\hline & \multicolumn{2}{|c|}{ PROFESOR } & ALUMNO \\
\hline
\end{tabular}

Tabla 2. Representación sinóptica de los procesos de enseñanza-aprendizaje y de la evaluación. (Castillo, 2002) 
En toda actividad de enseñanza-aprendizaje intencional, el profesorado programa qué va a enseñar y planifica cómo lo va a conseguir; y el discente, por su parte, intenta progresar en la dirección que le muestra su profesor con más o menos resistencia. Para que esta concurrencia de acciones tenga éxito debe existir una regulación de las acciones de enseñanza-aprendizaje. La evaluación es la pieza clave de la regulación del proceso (Castillo, 2002).

El objeto prioritario de la evaluación es el progreso educativo de los estudiantes porque esa es la finalidad del proceso de enseñanza-aprendizaje, pero no hay que olvidar que la persona es una realidad ampliamente compleja, y que reunir datos significativos sobre su progreso para evaluarla no siempre es una tarea fácil. Una parte de la tarea de evaluar empieza en la definición de objetivos didácticos. Pero indudablemente el progreso educativo de los estudiantes es el objetivo natural de la evaluación.

La nueva evaluación formativa en la práctica se traduce en una acción interactiva que aporta al estudiante información necesaria para hacer las correcciones en su aprendizaje y el docente para realizar cambios en las condiciones de aprendizaje. La evaluación formativa en todos los casos pretende enriquecer y mejorar las actuaciones futuras del estudiante.

Bloom (1969) pone énfasis en la evaluación formativa como elemento efectivo para el proceso tanto de enseñanza como de aprendizaje, recomendando su uso frecuente, Coll (1983) reafirma el valor regulador de la evaluación formativa para proporcionar información sobre el proceso de enseñanza y aprendizaje con fines de reajuste. En general, se resalta la evaluación formativa como un proceso que debiera ser dirigido y efectuado por el docente.

Para los constructivistas el error es un elemento altamente positivo para producir tipos de perturbaciones que crean el necesario desequilibrio para la autorreflexión y reestructuración conceptual. No existen respuestas malas sino respuestas que permitan al docente asumir la función de mediador que ayude al estudiante a construir sus propias estructuras de conocimiento. Ya lo decía Stenhouse (1984), evaluar es comprender, y obviamente la comprensión conduce al cambio.

Como también que el examen no garantiza que aquello que se pregunta sea lo más relevante. La tarea prioritaria del docente es orientar el aprendizaje y asegurar que aquello que se aprende es valioso y merece dedicarle tiempo y esfuerzo. El docente tiene que actuar como un crítico reflexivo y no sólo como calificador. Su función es la enseñanza de la autoevaluación en el discente.

Si se sitúan en el plano de un símil deportivo. El estudiante-atleta que quiere desarrollar sus cualidades físico-deportivas, e incluso ir superando sus propias marcas y llegar a competir en un estadio, tendrá en su docente el entrenador-preparador que va a intentar sacar lo mejor de sus posibilidades. Ello supone un conocimiento minucioso del discente-atleta (evaluación inicial-diagnóstica); y muchas horas de entrenamiento (horas lectivas), propiciando el desarrollo y corrigiendo errores (evaluación formativa 
o reguladora). Pero el día de la prueba en el estadio, el profesor-entrenador se convierte en juez de la misma, a falta de otra persona ajena (evaluación final-sumativa). En otras palabras, el profesor mediante la evaluación busca unos objetivos formativos en sus alumnos/as en todo momento: al inicio, durante el proceso de entrenamiento y al final. Aunque siempre es preferible un profesor-entrenador (preparador y facilitador), a un profesor-juez que sanciona el resultado, para atender a la realidad personal y social del alumno: son necesarios los dos.

Sin embargo hay que reconocer, lamentablemente, que en la práctica diaria de muchas aulas y de muchos centros, la evaluación sigue siendo la hora del examen, el día del examen o la semana de exámenes. No se trata de denunciar o de responsabilizar a nadie de esta situación. Es un cambio de la conceptualización de la evaluación, en particular, y de la acción didáctica, en general, que debe ir calando en todos los responsables más directamente implicados: Inspección, directores de centros educativos, docentes, discentes, padres, madres....Sólo cuando se produzca este cambio de mentalidad se irá abriendo paso a una nueva cultura de evaluación.

\section{Método}

La investigación, respecto a la generalización de resultados, es activa dado que tiene como fin solucionar los problemas que tiene el alumnado en aspectos relacionados con la evaluación en un determinado contexto.

La finalidad de la investigación es la construcción de un modelo para comprender los procesos de ases en la evaluación desde la perspectiva del alumnado. Un proyecto aplicado dado que se plantea la resolución de problemas a través del diseño de un modelo de formación bajo la perspectiva de la Investigación Acción Colaborativa (IAC)

La metodología seguida en la investigación la enmarcamos desde la superación del paradigma cuantitativo y se desarrolla utilizando el método de encuesta, a través de un cuestionario dirigido a discentes, y así obtener la información necesaria para describir dichas necesidades.

El instrumento elegido ha sido el cuestionario como técnica de recogida de datos.

El método de encuesta es el más apropiado en las disciplinas sociales, surge en la antigüedad a través de las enseñanzas de Sócrates, y su objetivo fundamental es la recogida de información sobre un tema concreto.

Los estudios basados en el método de encuesta que, como en este caso, se apoyan en el uso de cuestionarios facilitan la exploración (una descripción y explicación primeras) de una determinada problemática educativa.

En la investigación que se presenta, consideramos los cuestionarios, como buenos instrumentos metodológicos para la deliberación profesional crítica de discentes. Permite obtener una cualificada información de y en la propia realidad de la actividad práctica, examinar sistemáticamente y de un modo interactivo, los factores y variables 
que la condicionan; algunas investigaciones, sin embargo, incluyen la comparación de dos o más casos (Wittrock, 1989).

Los objetivos que se pretenden conseguir son:

- Describir la evaluación que se hace del proceso de aprendizaje.

- Ofrecer al profesorado de educación secundaria información para conocer, reflexionar, analizar, aplicar y desarrollar de forma responsable y autónoma la evaluación realizada por los estudiantes.

- Estimular la potencialidad formativa y la incidencia de la evaluación en los estudiantes.

- Conocer la percepción que tienen los estudiantes respecto de la evaluación de su aprendizaje.

Una vez preparado el instrumento se ha procedido a llenado por parte de los estudiantes. Una vez en los institutos se procedió a explicar en qué consistía. Una vez recogidos los cuestionarios, fueron volcados en una base de datos para su posterior análisis, que se realizó mediante el programa estadístico SPSS versión 20.

Hemos seleccionado el primer curso de bachillerato de los Institutos de Educación Secundaria "María Bellido" de Bailén y "Jabalcuz" de Jaén capital por la disponibilidad, posibilidades de acceso a los centros y el número de estudiantes. La población de la investigación ha sido de 180 estudiantes, la muestra final ha sido de 128, repartidos de la siguiente forma:

\begin{tabular}{|l|r|r|}
\hline & Frecuencia & Porcentaje \\
\hline NS/NC & 2 & 1,6 \\
Bailén (IES María & 100 & 78,1 \\
Bellido) & & \\
Jaén (IES Jabalcuz) & 26 & 20,3 \\
Total & 128 & 100,0 \\
\hline
\end{tabular}

Tabla 3. Ítem x76 (Instituto) 


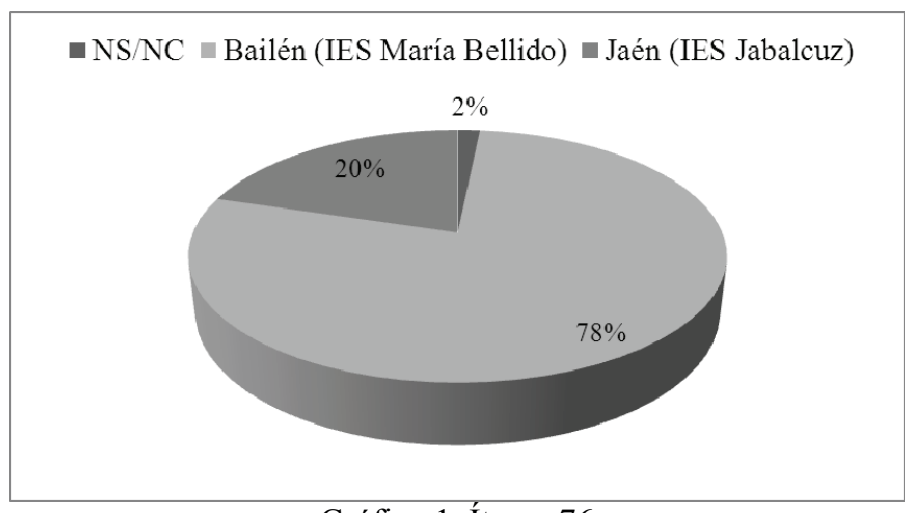

Gráfico 1. Ítem x76

El cuestionario ha sido rellenado por los estudiantes en la primera semana del mes de junio de 2013, una vez que fue validado por cinco expertos en educación seleccionados y pertenecientes a diferentes niveles educativos. Siguiendo a Selltiz (1980:722), en la elaboración de uno de los cuestionarios pilotos, tuvimos en cuenta las siguientes etapas:

a) Decisión sobre qué información debe buscarse.

b) Tipo de cuestionario que se va a utilizar.

c) Redacción de un primer borrador.

d) Revisión de las preguntas.

e) Comprobación previa de los cuestionarios.

f) Revisión y determinación de procedimientos para su utilización.

De este modo, el cuestionario para discentes definitivo quedó configurado una vez revisadas dichas etapas.

Distribuidos los cuestionarios y recogidas las respuestas, se ha procedido al análisis factorial exploratorio. Los siete factores que se han identificado explican la varianza y reflejan una estructura en la que se destacan con cierta nitidez algunas dimensiones.

Por tanto, la estructura observada hace referencia a que las diferentes dimensiones del cuestionario más que independientes están relacionadas entre sí; idea ésta que ha presidido la construcción del cuestionario. El estudio de la fiabilidad (medida por el coeficiente Alfa de Cronbach) para cada dimensión corrobora la idea de relación entre las dimensiones. Los valores bajos obtenidos indican que existe baja consistencia entre los ítems que forman cada dimensión. Es decir, los indicadores (ítems) considerados para medir un constructo (por ejemplo, evaluación) no son considerados por los discentes como pertenecientes necesariamente a ese constructo; de hecho, los ítems pertenecerían a dos o más dimensiones que no son conceptualmente independientes.

Para determinar qué ítems son pertinentes y cuáles deben descartarse, se han considerado los índices de discriminación u homogeneidad y de validez de los ítems. 
Estos, junto con el análisis descriptivo de las respuestas, han aconsejado la eliminación o modificación de determinadas opciones.

La fiabilidad del instrumento se ha obtenido a través del paquete estadístico SPSS 19.0, obteniéndose un índice de correlación igual a $0^{\prime} 83$ (Tabla 4), lo que indica una correlación alta próxima a 1, que sería la correlación perfecta. Como indica Fox (1987) a la hora de estimar respuestas, son aceptables correlaciones a partir de $0^{\prime} 70$ e incluso de $0^{\prime} 60$, cuando se realizan estimaciones de opinión y crítica, como en el caso de la presente investigación.

\begin{tabular}{c|c|c}
\hline \multicolumn{3}{c}{ Estadísticos de fiabilidad } \\
\hline $\begin{array}{c}\text { Alfa de } \\
\text { Cronbach }\end{array}$ & $\begin{array}{c}\text { Alfa de Cronbach basada en } \\
\text { los elementos tipificados }\end{array}$ & N de elementos \\
\hline, 833 &, 840 & 67 \\
\hline
\end{tabular}

Tabla 4. Alfa de Cronbach

El total de 33 ítems del cuestionario se distribuían formalmente en una serie de dimensiones, que quedan recogidas en la siguiente tabla.

\begin{tabular}{cc}
\hline EJES/DIMENSIONES & ITEMS \\
\hline CONCEPTO DE EVALUACIÓN & 2 \\
\hline $\begin{array}{c}\text { CONCEPTO DE EVALUACIÓN DEL } \\
\text { PROFESOR/A }\end{array}$ & \\
\hline
\end{tabular}

INSTRUMENTOS DE EVALUACIÓN 3

SISTEMAS METODOLÓGICOS 6-8,17,19,22,31

RELACIÓN CON EL AULA/

ENSEÑANZA-APRENDIZAJE

$1,4,15-16,25$ 


\begin{tabular}{cc}
\hline MOMENTO DE EVALUACIÓN & $5,18,27-28$ \\
\hline ASESORAMIENTO DEL DOCENTE & $9-14,19-21,23-24,26,29,30,32$ \\
\hline HABILIDADES DEL DOCENTE & 33 \\
\hline
\end{tabular}

Tabla 5. Dimensiones del Cuestionario (Fuente: Elaboración propia)

En cuanto a la validación de los cuestionarios por parte de los expertos, participaron especialistas de la Universidad de Jaén, Centro de Profesores de Linares-Andújar, Equipo de Orientación Educativa de Bailén, alumnado del I.E.S. "Hermanos Medina Rivilla" de Bailén. Se les entregó una ficha registro, con el fin de valorar la pertinencia y ubicación de cada uno de los ítems, lo que permitió su revisión. Se planteó a los expertos la conveniencia de la ubicación de los ítems, alternativas a la misma, lenguaje, entre otros, Torres (1996:218).

Por último, se le plantearon una serie de preguntas abiertas sobre los cuestionarios globalmente:

- A su juicio, ¿qué otros aspectos debería incluir?

- ¿Debería suprimirse alguno de los ítems?

- Valore la estructura del cuestionario.

- ¿Considera adecuado el formato del cuestionario, a la hora de cumplimentarlo? Justifíquelo.

- ¿Qué otros aspectos considera, podían incluirse?.

El cuestionario se ha quedado agrupado en las siguientes dimensiones que pretenden estudiar la naturaleza multifactorial del proceso evaluador. Dichas dimensiones quedan recogidas en la tabla 6.

\begin{tabular}{|c|c|c|c|}
\hline $\begin{array}{l}\text { Tipo de } \\
\text { Ítem }\end{array}$ & $\begin{array}{l}\text { Escala de } \\
\text { medición }\end{array}$ & Información que pretendemos recoger & Dimensión \\
\hline $\mathrm{x}_{01}$ & Likert & Necesidades para estudios posteriores. & \multirow{4}{*}{$\begin{array}{l}\text { Planteamiento } \\
\text { de la enseñanza } \\
\text { según el } \\
\text { profesor }\end{array}$} \\
\hline $\mathrm{x}_{02}$ & Likert & Sabe la mayoría de los estudiantes. & \\
\hline $\mathrm{x}_{03}$ & Likert & Sabe cada discente. & \\
\hline $\mathrm{x}_{04}$ & Likert & No saben nada. & \\
\hline $\mathrm{x}_{05}$ & Likert & Poner calificación. & \multirow[b]{2}{*}{ Opinión sobre } \\
\hline $\mathrm{x}_{06}$ & Likert & Detectar avance. & \\
\hline
\end{tabular}




\begin{tabular}{|c|c|c|c|}
\hline $\mathrm{x}_{07}$ & Likert & Emitir diagnóstico. & \multirow{3}{*}{$\begin{array}{l}\text { evaluación del } \\
\text { profesor. }\end{array}$} \\
\hline $\mathrm{x}_{08}$ & Likert & Recoger datos. & \\
\hline $\mathrm{x}_{09}$ & Likert & Tener información. & \\
\hline $\mathrm{x}_{11}$ & Likert & Pruebas objetivas. & \multirow{6}{*}{$\begin{array}{l}\text { Instrumentos de } \\
\text { evaluación }\end{array}$} \\
\hline $\mathrm{x}_{12}$ & Likert & Pruebas de respuesta libre. & \\
\hline $\mathrm{x}_{13}$ & Likert & Cuadernos. & \\
\hline $\mathrm{x}_{14}$ & Likert & Casos prácticos. & \\
\hline $\mathrm{x}_{15}$ & Likert & Oral. & \\
\hline $\mathrm{x}_{16}$ & Likert & Trabajos. & \\
\hline $\mathrm{x}_{17}$ & Likert & No es necesario. & \multirow{4}{*}{$\begin{array}{l}\text { Conocimientos } \\
\text { relacionados } \\
\text { con la práctica } \\
\text { en la clase }\end{array}$} \\
\hline $\mathrm{x}_{18}$ & Likert & Es necesario. & \\
\hline $\mathrm{x}_{19}$ & Likert & Conocimiento importante por sí mismo. & \\
\hline $\mathrm{x}_{20}$ & Likert & $\begin{array}{l}\text { Conocimientos tienen que tener aplicación } \\
\text { práctica. }\end{array}$ & \\
\hline $\mathrm{x}_{21}$ & Likert & Trimestre. & \multirow{4}{*}{$\begin{array}{l}\text { Momento para } \\
\text { mejorar las } \\
\text { prácticas } \\
\text { evaluadoras }\end{array}$} \\
\hline $\mathrm{x}_{22}$ & Likert & Final de curso. & \\
\hline $\mathrm{x}_{23}$ & Likert & Demanda. & \\
\hline $\mathrm{X}_{24}$ & Likert & Fases de evaluación. & \\
\hline $\mathrm{x}_{25}$ & Likert & \multicolumn{2}{|c|}{ Criterios de evaluación corresponden a los objetivos. } \\
\hline $\mathrm{X}_{28}$ & Likert & \multicolumn{2}{|l|}{ Asesoramiento en grupo. } \\
\hline $\mathrm{X}_{29}$ & Likert & \multicolumn{2}{|l|}{ Asesoramiento individual. } \\
\hline $\mathrm{x}_{30}$ & Likert & \multicolumn{2}{|l|}{ Adaptación del asesoramiento cada año. } \\
\hline $\mathrm{x}_{31}$ & Likert & \multicolumn{2}{|l|}{ Asesomiento mejora el proceso E-A. } \\
\hline $\mathrm{x}_{32}$ & Likert & \multicolumn{2}{|l|}{ Asesoramiento mejora la evaluación. } \\
\hline $\mathrm{x}_{33}$ & Likert & \multicolumn{2}{|l|}{$\begin{array}{l}\text { Asesoramiento-formación, mejora de la evaluación de los } \\
\text { estudiantes. }\end{array}$} \\
\hline $\mathrm{x}_{34}$ & Likert & \multicolumn{2}{|l|}{ Asesoramiento reflejado en clase. } \\
\hline $\mathrm{x}_{35}$ & Likert & \multicolumn{2}{|l|}{ Mejora de las pruebas al realizar evaluación. } \\
\hline $\mathrm{X}_{36}$ & Likert & \multicolumn{2}{|l|}{ Profesor valora los cambios de la evaluación. } \\
\hline $\mathrm{x}_{39}$ & Likert & \multicolumn{2}{|l|}{ Puesta en marcha de sugerencias de asesoramiento. } \\
\hline $\mathrm{x}_{40}$ & Likert & \multicolumn{2}{|l|}{ Adquisición de hábitos y técnicas. } \\
\hline $\mathrm{x}_{41}$ & Likert & Comprender la realidad del asesoramiento. & \multirow{3}{*}{$\begin{array}{l}\text { Intención del } \\
\text { asesoramiento } \\
\text { del docente }\end{array}$} \\
\hline $\mathrm{x}_{42}$ & Likert & $\begin{array}{l}\text { Desarrollo del pensamiento crítico y autónomo } \\
\text { del asesor. }\end{array}$ & \\
\hline $\mathrm{x}_{43}$ & Likert & Servir para el día a día. & \\
\hline $\mathrm{x}_{45}$ & Likert & Recoger las expresiones de docentes. & \multirow{4}{*}{$\begin{array}{l}\text { Necesidad de } \\
\text { asesoramiento a } \\
\text { su profesor }\end{array}$} \\
\hline $\mathrm{x}_{46}$ & Likert & Intercambiar puntos de vista. & \\
\hline $\mathrm{x}_{47}$ & Likert & Evaluación conjunta. & \\
\hline $\mathrm{X}_{48}$ & Likert & Punto de encuentro de docentes. & \\
\hline $\mathrm{X}_{49}$ & Likert & Promoción de la carrera. & \multirow{4}{*}{$\begin{array}{l}\text { Consecuencias } \\
\text { del } \\
\text { asesoramiento } \\
\text { en el profesor. }\end{array}$} \\
\hline $\mathrm{x}_{50}$ & Likert & Mejoras en las retribuciones económicas. & \\
\hline $\mathrm{x}_{51}$ & Likert & Algún premio. & \\
\hline $\mathrm{x}_{52}$ & Likert & Mejora servicio profesional. & \\
\hline $\mathrm{x}_{53}$ & Likert & \multicolumn{2}{|l|}{ Docente y animación de colegas. } \\
\hline $\mathrm{x}_{54}$ & Likert & \multicolumn{2}{|l|}{ Voluntario. } \\
\hline $\mathrm{x}_{55}$ & Likert & \multicolumn{2}{|l|}{ Obligatorio. } \\
\hline $\mathrm{X}_{56}$ & Likert & Horas lectivas. & \\
\hline $\mathrm{x}_{57}$ & Likert & Vacaciones. & \\
\hline $\mathrm{X}_{58}$ & Likert & Fuera del horario lectivo pero retribuido. & Mlodos de \\
\hline $\mathrm{X}_{59}$ & Likert & Consecuencias para la promoción. & \\
\hline $\mathrm{x}_{61}$ & Likert & Colaboración entre colegas sobre evaluación. & \\
\hline
\end{tabular}




\begin{tabular}{|c|c|c|}
\hline $\mathrm{x}_{62}$ & Likert & Eficacia de la evaluación de los profesores. \\
\hline $\mathrm{x}_{65}$ & Likert & Habilidades del profesor para crear un clima propicio. \\
\hline $\mathrm{x}_{66}$ & Likert & Etapa dónde se necesita mayor asesoramiento en la evaluación. \\
\hline $\mathrm{x}_{67}$ & Likert & Motivación. \\
\hline $\mathrm{x}_{68}$ & Likert & Gestión. \\
\hline $\mathrm{x}_{69}$ & Likert & Reflexión. \\
\hline $\mathrm{x}_{70}$ & Likert & Relaciones. \\
\hline $\mathrm{x}_{71}$ & Likert & Conflictos \\
\hline $\mathrm{x}_{72}$ & Likert & Habilidades del \\
\hline $\mathrm{x}_{73}$ & Likert & profesorado \\
\hline $\mathrm{x}_{74}$ & Likert & Disponibilidad. \\
\hline
\end{tabular}

\begin{tabular}{cll}
\hline $\begin{array}{c}\text { Tipo de } \\
\text { Item }\end{array}$ & Escala de medición & \multicolumn{1}{c}{ Información que pretendemos recoger } \\
\hline $\mathrm{y}_{10}$ & Abierta 1 & Su visión de la evaluación. \\
\hline $\mathrm{y}_{26}$ & Abierta 2 & Mejor forma de capacitar a docentes. \\
\hline $\mathrm{y}_{27}$ & Abierta 3 & Qué puede mejorar el docente. \\
\hline $\mathrm{y}_{38}$ & Abierta 4 & Criterios para el asesoramiento docente. \\
\hline $\mathrm{y}_{44}$ & Abierta 5 & Influencia de la evaluación en la educación del discente. \\
\hline $\mathrm{y}_{60}$ & Abierta 6 & Otras propuestas sobre los modos de asesoramiento. \\
\hline $\mathrm{y}_{63}$ & Abierta 7 & Asesoramiento al profesorado en la evaluación. \\
\hline $\mathrm{y}_{64}$ & Abierta 8 & Mejor asesoramiento al docente en la evalluación de discentes. \\
\hline $\mathrm{y}_{75}$ & Abierta 9 & Otras habilidades del docente. \\
\hline
\end{tabular}

\begin{tabular}{|c|c|c|}
\hline $\begin{array}{c}\text { Tipo de } \\
\text { Item }\end{array}$ & Escala de medición & Información que pretendemos recoger \\
\hline $\mathrm{Z}_{37}$ & Dicotómica & Acuerdo sobre la evaluación de la enseñanza del profesor \\
\hline
\end{tabular}

Tabla 6. Dimensiones del Cuestionario agrupado por ítems (Fuente: Elaboración propia)

Desde la tradición interpretativa, Pérez (1990), plantea el gran impulso que se ofrece al análisis de la realidad desde este planteamiento de investigación. En este modelo los prácticos participan en la investigación a diferencia del paradigma positivista permitiendo la aproximación a la realidad. Este paradigma nos va a permitir comprender mejor las distancias que se presenten entre la teoría y el mundo de la práctica, Shulman, (1986). Este paradigma estaría próximo a corrientes de tipo antropológico, sociológico, psicológico, como la metodología cualitativa, la investigación etnográfica, etc.

No aspira el paradigma interpretativo a la generalización, porque cada proceso es único. La objetividad hay que buscarla en la intersubjetividad de los investigadoresdocentes, que viven el problema, constituyendo los informes de la investigación hipótesis a contrastar en contextos semejantes, Pérez (1990).

Desde el paradigma IAC, nos planteamos que en un contexto de Educación Secundaria Obligatoria, donde los propios discentes van construyendo sus propios aprendizajes en un proceso continuo de interiorización de los mismos, el modelo que más responde a la investigación estaría próximo al modelo de investigación acción 
colaborativa, desde el cual se plantea el trabajo conjunto y la interacción progresiva entre investigador y prácticos, Pérez (1990:153).

Medina (1989), por su parte, diferencia la perspectiva socio-crítica o de formación de la acción reflexiva, cuyo marco de investigación es el paradigma ecológico-crítico. El eje de la formación se centra en la formación del docente en la reflexión crítica en torno a su concepción y práctica de la evaluación.

\section{Resultados}

Este es el análisis que se hace de los resultados. Presentamos cada dimensión e ítem del cuestionario. En este formulario donde han participado 128 estudiantes que han respondido a los 33 ítems del cuestionario.

La dimensión 1 nos lleva a conocer como cree el discente que plantea los docentes la enseñanza de su asignatura. Un $62.5 \%$ esta de acuerdo teniendo en cuenta muy claramente las necesidades para estudios posteriores (Likert 01), el 39'8\% dice estar también de acuerdo con tener en cuenta lo que sabe de la asignatura la mayoría de los estudiantes (Likert 02), un 38'3\% le resulta indiferente con tener en cuenta lo que sabe cada uno de ellos (Likert 03) y el 28'9\% mantiene estar de acuerdo con partir de la base de que no saben nada ya que todo se olvida (Likert 04).

La dimensión 2 nos informa que el estudiante está de acuerdo en un 42 '2\% que evaluar para su profesor/a es cumplir con la obligación de poner una calificación (Likert 05); un 53'9\% está de acuerdo en que la evaluación que realiza su profesor/a sirve para detectar el avance de los estudiantes respecto a su punto de partida (Likert 06); el 39'8\% le resulta indiferente que sirva para emitir un diagnóstico para la orientación (Likert 07); para la gran mayoría de los estudiantes participantes en la investigación, un 39'1\% le resulta indiferente que sirva para recoger datos para replantear la programación (Likert 08), al igual que también le resulta indiferente, con un $34^{\prime} 4 \%$, que sirva para tener información para plantear la recuperación.

En esta dimensión hemos planteado una pregunta abierta, un $43 \%$ ha opinado no saber qué es la evaluación.

La dimensión 3 recoge información sobre los sistemas metodológicos que utilizan los docentes y que son valorados por los estudiantes.

Un 52'3\% considera estar de acuerdo que los criterios de evaluación responden a los que llevan a cabo el profesorado con los objetivos propuestos y los contenidos desarrollados (Likert 25); el 41'4\% afirma que a menudo su profesor sabe crear un clima propicio para la evaluación y la negociación de los resultados (Likert 65).

Con un $32 \%$ opinan que bastantes veces el profesorado valora positivamente los cambios que ha venido realizado en la evaluación (Likert 36). 
Las siguientes preguntas de la dimensión son abiertas. Un 35'2\% del alumnado opina que las opiniones que reciba el profesorado les parece la mejor forma de capacitación para que sus docentes aprendan a evaluarse su enseñanza (Likert 26). A la pregunta "sabe en qué puede mejorar sus profesores/as para llevar a cabo la evaluación de los procesos de aprendizaje de los estudiantes", hay diversidad de opiniones, un $18 \%$ no sabe o no contesta a la pregunta, seguida muy de cerca por la observación del trabajo en la clase con un $17^{\prime} 2 \%$ (Likert 27).

También existe diversidad de opiniones cuando se les pregunta "en qué medida o forma cree que puede influir la evaluación en la Educación que reciben", el 28'1\% no sabe que contestar y el 22'7\% cree que puede ser en la motivación (Likert 44). En cuanto a "los criterios que debe tener en cuenta para el asesoramiento de su docente", un $45^{\prime} 3 \%$ desconoce esos criterios, aunque consideran con un $19^{\prime} 5 \%$ que es importante tomar en cuenta la participación de los estudiantes en clase (Likert 38).

La dimensión 4 se caracteriza por la relación con el aula/enseñanza-aprendizaje. Un 62 ' $5 \%$ está de acuerdo que el docente plantea la enseñanza de su materia teniendo en cuenta muy claramente las necesidades para estudios posteriores (Likert 01), mientras que un $39^{\prime} 8 \%$ está de acuerdo que es teniendo en cuenta lo que ya sabe de la asignatura la mayoría de los estudiantes (Likert 02). A una amplia mayoría, 38'3\%, considera indiferente que su profesor/a platee la enseñanza de su asignatura teniendo en cuenta lo que sabe cada estudiante (Likert 03) frente a un $28^{\prime} 9 \%$ está en desacuerdo que su profesor plantea la enseñanza de su asignatura partiendo de la base de que no saben nada ya que todo se olvida (Likert 04).

Un $34 \% \%$ está en total desacuerdo que los conocimientos que imparte su docente deben estar relacionados con su práctica en la clase ya que no es necesario, si el estudiante lo entiende ya lo aplicará (Likert 17), un 54'7\% está de acuerdo que es totalmente necesario; ya que servirá como motivación (Likert 18). Al 40'6\% le resulta indiferente porque no es necesario, el conocimiento es importante en sí mismo (Likert 19 ), frente a un $56^{\prime} 3 \%$ que está totalmente de acuerdo en que es totalmente necesario, los conocimientos tienen que tener siempre práctica en la clase (Likert 20).

Un $52 ' 3 \%$ opina que algunas veces que el asesoramiento recibido por su docente acerca de la evaluación se ve reflejado en el trabajo en las clases (Likert 34). Al igual, un $50{ }^{\prime} 8 \%$ considera que algunas veces han mejorado las pruebas que aplica su profesor/a en clase al realizar la evaluación del aprendizaje de los estudiantes (Likert 35).

Un $35^{\prime} 2 \%$ opina que a menudo su profesor/a anima a otros docentes del centro para el desarrollo de actividades de asesoramiento relacionadas con la evaluación (Likert 53).

La dimensión 5 ofrece información sobre el momento de evaluación. Un 50\% está totalmente de acuerdo que el mejor momento o período para que sus profesores mejoren sus prácticas evaluadoras es cada trimestre (Likert 21), el 22'7\% le resulta indiferente si es al final de curso (Likert 22), igual que el 41'1 que opina indiferente si es cuando el profesorado lo demande (Likert 23) y a un $39^{\prime} 1 \%$ también le resulta indiferente si se debe alcanzar las fases de la evaluación (Likert 24). 
El $89^{\prime} 8 \%$ opina que el profesorado debe de realizar la evaluación de su enseñanza (Likert 37).

El 45'3\% opina que a menudo cree que existe colaboración en tareas de evaluación entre sus profesores/as (Likert 61), mientras que un 46'1\% considera algo eficaz la evaluación de sus profesores/as en las asignaturas que imparten (Likert 62).

La dimensión 6, con más ítems del cuestionario, está relacionada con el asesoramiento del docente.

Un $35^{\prime} 9 \%$ considera estar de acuerdo para que el asesoramiento que recibe el profesorado sea en grupo (Likert 28). El 29'7\% considera indiferente que dicho asesoramiento para el profesorado se realice de forma individual (Likert 29).

Bastantes veces, un $41^{\prime} 4 \%$, opina que el asesoramiento debería de adaptarse cada año escolar en la asignatura que imparte cada docente (Likert 30). Un 48'4\% argumenta que el asesoramiento que recibe el docente es importante para la mejora del proceso de enseñanza-aprendizaje en la clase (Likert 31).

El $50{ }^{\prime} 8 \%$ da bastante importante para que el asesoramiento que recibe su docente mejore la evaluación (Likert 32).

Bastantes veces, $40^{\prime} 6 \%$, cree el alumnado participante que el asesoramiento y formación que ha recibido su docente le han permitido mejorar la evaluación de los estudiantes (Likert 33).

El $45^{\prime} 3 \%$, opina no saber los criterios que se deben tener en cuenta para el asesoramiento del profesorado (Likert 38).

Un $48^{\prime} 4 \%$ reflexiona y opina que algunas sugerencias de asesoramiento formuladas por otros docentes pone en marcha su profesor/a (Likert 39).

El 63'3\% opina estar de acuerdo que sus docentes con el asesoramiento que reciben creen que intentan conseguir hábitos y técnicas para una mejor evaluación (Liket 40), un $53^{\prime} 1 \%$ también está de acuerdo pues sirve para adquirir conocimientos que les permitan comprender la realidad del asesoramiento (Likert 41). Sin embargo, un $36^{\prime} 7 \%$ considera indiferente que sirva para desarrollar un pensamiento crítico y autónomo del asesor (Likert 42) y un 36 $7 \%$ aprecia que ese asesoramiento le sirva para el día a día (Likert 43).

Un 48'4\% considera estar de acuerdo que el asesoramiento a sus profesores/as es necesario para que se recojan más fácilmente las expresiones de todos los docentes del grupo (Likert 45); el 54'7\% aprecia estar de acuerdo que sea para intercambiar puntos de vista a fin de obtener una valoración conjunta (Likert 46); un 38'3\% también está de acuerdo que el asesoramiento sirva para realizar una evaluación conjunta (Likert 47), mientras que el $35^{\prime} 9 \%$ se posiciona indiferente para que sirva de punto de encuentro entre todo el profesado (Likert 48). 
En cuanto a las consecuencias que debería tener el asesoramiento al profesorado en la evaluación, un $39^{\prime} 8 \%$, se muestra indiferente que sirva para la promoción en la carrera del docente (Likert 49); el 42'2\% también se muestra indiferente para la mejora de las retribuciones económicas (Likert 50); Otro porcentaje importante, el 37'5, se muestra indiferente que la consecuencia sea algún tipo de premio (Likert 51) y un $51^{\prime} 6 \%$ está de acuerdo que sirva para ayudar a que mejore el ejercicio profesional del docente (Likert 52).

El alumnado participante en el cuestionario considera que el asesoramiento que reciba su profesor/a, están de acuerdo, en un 38"3\% que debería de ser voluntario (Likert 54), además un 37'5\% también está de acuerdo que no importa el cómo pero sí con consecuencias para la promoción (Likert 59). Sin embargo, el 24'2\% (Likert 55), el 39'1\% (Likert 56), el 30'5\% (Likert 57) y el 44'5\% (Likert 58), respectivamente, opinan de forma indiferente en cuanto a que sea obligatorio, en horas lectivas, en las vacaciones o fuera de la jornada escolar pero retribuidas, respectivamente.

Encontramos que un $55^{\prime} 5 \%$ de los encuestados no sabe sugerir otras propuestas sobre el asesoramiento que debe recibir su profesorado (Likert 60), la primera sugerencia con un $15^{\prime} 6 \%$ es que sea con ejemplos.

Un $27 \% 3 \%$ considera que personal cualificado (inspectores, asesores de CEP, profesorado universitario, etc) son quienes deben de asesorar al profesorado en la evaluación (Likert 63).

El análisis de cada caso es lo que opina una amplia mayoría, el 46'1\%, para indicar que es el mejor asesoramiento que le pueden ofrecer a sus docentes para abordar la evaluación de los estudiantes (Likert 64).

El 42'2\% considera que la Educación Secundaria es la etapa educativa donde el profesorado necesita un mayor asesoramiento en la evaluación (Likert 66).

La dimensión 7 muestra la opinión del alumnado de las habilidades de sus docentes.

Un $54^{\prime} 7 \%$ considera de manera media que sus docentes están motivados por el trabajo (Likert 67), otro 53'1\% opina que también es media la gestión del trabajo que realizan (Likert 68). Llama la atención que un 39'8\% considera media el propiciar la reflexión en clase, mientras que un 39'1\% considera que es baja (Likert 69).

Un 40'6\% considera baja las relaciones interpersonales (apertura, saber escuchar, apoyo personal) (Likert 70), al igual que también opinan bajo de la resolución de conflictos con un $38^{\prime} 3 \%$ (Likert 71).

Un $45^{\prime} 3 \%$ considera medio la habilidad de diagnóstico individual y organizativo (Likert 72), otro 39' $8 \%$ considera medio el propiciar apoyo y recursos (Likert 73) y un $41^{\prime} 4 \%$ opina que es media la habilidad del docente para la disponibilidad para atender al alumnado (Likert 74). Sin embargo, el 92'2\% no indica ninguna otra habilidad que aprecien en sus docentes. 


\section{Discusión}

A raíz de los resultados llegamos a las siguientes conclusiones para la mejora de la evaluación en los institutos de educación secundaria, desde la perspectiva de los estudiantes:

- La mayoría del alumnado cree que su profesor/a plantea su asignatura teniendo en cuenta muy claramente las necesidades para estudios posteriores y no lo que sabe el discente de la asignatura o partiendo de la base de que no saben nada porque se les olvida todo.

- Piensan que la evaluación que realizan los docentes sirve para cumplir con la obligación de emitir una calificación.

- Los estudiantes relacionan por orden, de mayor a menor valoración, los instrumentos de evaluación que sus profesores/as utilizan en clase:

- Pruebas objetivas.

- Trabajos o proyectos realizados por los discentes.

- Cuadernos de los estudiantes.

- Problemas/casos prácticos.

- Pruebas de respuesta libre o abiertas.

- Preguntas orales.

- Una mayoría considera que sus profesores/as deben de impartir conocimientos que estén relacionados con la práctica en la clase.

- Los estudiantes razonan que sus profesores deben recibir asesoramiento para mejorar sus prácticas evaluadoras cada trimestre y no a final de curso, cuando lo demanden o se produzcan las sesiones de evaluación.

- En cuanto a si los criterios de evaluación que llevan a cabo los docentes se corresponden con los objetivos y contenidos propuestos, los discentes no saben muy bien qué quiere decir la pregunta, no porque no sepan contestarla sino porque ellos consideran que el objetivo de su profesor es el de que aprueben la asignatura.

- Los estudiantes opinan que los docentes deben de tener muy en cuenta a la hora de llevar a cabo la evaluación del discente:

- Valorar el trabajo diario y actitud de los alumnos.

- Conocer las capacidades de los alumnos.

- No ir demasiado rápido en las explicaciones.

- No tener prejuicios de los alumnos.

- Utilizar actividades prácticas de la asignatura.

- A los estudiantes le resulta indiferente si el asesoramiento de sus profesores/as debe ser en grupo o de forma individual.

- Consideran que el asesoramiento y formación hasta ahora recibido de su profesor/a no le ha permitido mejorar en sus evaluaciones.

- Los docentes deben de realizar la evaluación de su enseñanza. 
- La manera o forma de explicar del docente y la comprensión hacia el alumnado son los principales criterios donde debe de asesorarse el docente, según especifica el estudiante.

- Los estudiantes ven de manera positiva que el profesorado reciba asesoramiento.

- La evaluación que hace el docente de alumno/a influye mucho en la educación de estos últimos.

- Las consecuencias que debería tener el asesoramiento a su profesor/a en la evaluación deben ser le resulta indiferente al alumnado.

- El asesoramiento debe ser voluntario por parte del docente. Como propuestas hacen referencia a:

- Crítica abierta del alumnado.

- Reuniones entre profesores/as.

- Asesoramiento obligatorio.

- "No duden tanto en poner las notas".

- Por orden de preferencia, los discentes expresan que el asesoramiento al profesorado debe ser impartido por:

- Orientador/a.

- Alumnos/as.

- Director, como representante de la Administración en el centro.

- Catedráticos de Universidad.

- Gobierno de la comunidad. Centros de Profesores.

- Autoevaluación.

- Consejo Escolar del centro.

- A menudo el docente sabe crear un clima propicio para la evaluación y la negociación de los resultados.

- La Educación Secundaria Obligatoria es la etapa educativa donde el alumnado considera que el profesorado necesita más asesoramiento.

- La motivación y gestión del trabajo, propiciar a la reflexión en clase, saber escuchar, solventar conflictos, diagnostico individual, propiciar apoyo-recursos y disponibilidad para atender al alumnado, tiene especial relevancia para los estudiantes.

Por todo lo expuesto, se llegan a las siguientes conclusiones generales:

- Pensar y diseñar las actividades de evaluación plenamente integradas en el proceso de enseñanza-aprendizaje. La evaluación no es algo distinto o posterior, sino integrado en el modelo a la enseñanza del profesor y al aprendizaje del estudiante.

- No confundir la evaluación con un examen; ni la evaluación continua, con exámenes continuos o más frecuentes. El examen es sólo uno de los muchos 
instrumentos de recogida de información al servicio de la evaluación. La evaluación es mucho más: es recoger información válida, es poder formular un juicio de valor y es tener la capacidad para tomar las decisiones.

- El objetivo de la evaluación no es sólo poner notas a los estudiantes. Antes de llegar a esa fase calificatoria o acreditativa del proceso evaluador, están las fases y funciones: diagnóstica, formativa, reguladora, motivadora, de diálogo, etc., con que la evaluación acompaña a todo el proceso de enseñanza-aprendizaje, y que afecta tanto al docente como al estudiante.

- No hay que defender que las pruebas objetivas son mejores que las preguntas abiertas y viceversa. Hay que saber seleccionar los instrumentos necesarios que pueden proporcionar la información más adecuada, para cada caso o situación, según cada circunstancia.

- La evaluación no nos sirve, como se viene atribuyendo a los exámenes, para determinar qué estudiantes fracasan y quiénes tienen éxito. La evaluación no se debe quedar ahí, sino que, por el contrario, debe aportar información suficiente que explique por qué se producen resultados en términos de éxito o de fracaso; y sobre todo, que fundamente las decisiones de tratamiento o de mejora a las situaciones de fracaso.

- La evaluación no debe ser responsabilidad exclusiva del docente. El proceso de evaluación, como el proceso de enseñanza-aprendizaje, es una actividad docente compartida entre el docente y el alumnado. El estudiante, a partir de los estudios de Primaria, ya va siendo responsable de sus actos. Ya es capaz de reconocer sus esfuerzos o su desinterés; sus aciertos o errores, etc. Es educativo poner al estudiante frente a sus responsabilidades, también en la evaluación, fomentando para ello la autoevaluación del alumno.

\section{Referencias Bibliográficas}

BLOOM, B. S. (1969). Some theoretical issues relating to educational evaluation. In R. W. Tyler (Ed.). Educational evaluation: new roles, new means: the 63rd yearbook of the National Society for the Study of Education (part II) (Vol. 69(2), pp. 26-50). Chicago, IL: University of Chicago Press.

CASTILLO, S. (2002): Compromisos de la Evaluación Educativa. Madrid: Pearson Educación.

COLL, C. (1983): La evaluación en el proceso de enseñanza-aprendizaje. En Cuadernos de Pedagogía, núm. 103-104, pp.13-17.

FOX, D. (1981) (1987). El proceso de investigación en educación. Pamplona: Eunsa.

LOE: Ley Orgánica 2/2006, de 3 de mayo, de Educación. BOE nº 106 (04-05-06)

LOCE: Ley Orgánica 10/2002, de 23 de diciembre, de Calidad de la Educación. BOE $\mathrm{n}^{\mathrm{o}} 307$ (24-12-02)

LODE: Ley Orgánica del Derecho a la Educación. (BOE: 4-7-85). 
LOGSE: Ley Orgánica 1/1990 de 3 de octubre, de Ordenación General del Sistema Educativo. BOE $n^{\circ} 238$ de 4 de octubre.

LOPEGCE: Ley Orgánica 9/1995, de 20 de noviembre, de la participación, la evaluación y el gobierno de los centros docentes.

MEDINA, A. (2013) (Coord.). Formación del profesorado. Actividades innovadoras para el dominio de las competencias docentes. Madrid: Ramón Areces.

MEDINA, A. (1989): La formación del profesorado en una sociedad tecnológica. Madrid: Cincel.

PÉREZ, E. (2013). La formación del profesorado para el fomento y desarrollo de las instituciones de educación superior. En B. Valenzuela (2013). Modelos y Prácticas para la mejora del proceso de enseñanza-aprendizaje universitario. México D.F.: Pearson Educación. pp. 73-86

PÉREZ, R. (1990): Diagnóstico, Evaluación y Toma de Decisiones. Madrid: Rialp.

RODRÍGUEZ, J.L. y Tejedor, F.J. (1997): Evaluación Educativa: Evaluación de los aprendizajes de los alumnos. Salamanca: Universidad de Salamanca.

SELLTIZ, L.S. (1980) (Coord.). Métodos de investigación en las relaciones sociales. Madrid: Rialp.

SHULMAN, L.S. (1986). Those who understand: knowledge growth in teaching. Educational Researcher, 15 (2), 4-14.

STENHOUSE, L. (1984): Investigación y desarrollo del curriculum. Madrid: Morata.

TORRES, J.A. (1996): La formación del profesor tutor como orientador. Jaén: Universidad de Jaén.

VILLAR, L.M. (1992) (Coord.). Desarrollo profesional centrado en la escuela. Granada: Universidad de Granada/Force.

VILLAR, L.M. y ALEGRE, O.M. (2012). Los portafolios electrónicos en el hemisferio de la evaluación auténtica. Madrid: Síntesis.

WITTROCK, (1989). La investigación de la enseñanza, II. Barcelona: Piados.

\section{Correspondencia con el autor}

Eufrasio PÉREZ NAVÍO

Universidad de Jaén

Departamento de Pedagogía

Área de Didáctica y Organización Escolar

Despacho 234, Edificio C5, Buzón nº19

Campus Las Lagunillas s/n.

23071, Jaén (España)

Teléfono: +34953212380

email: epnavio@ujaen.es 